\title{
Thermal deformation in a thin circular plate due to a partially distributed heat supply
}

\author{
N L KHOBRAGADE ${ }^{1}$ and K C DESHMUKH ${ }^{2}$ \\ ${ }^{1}$ Department of Mathematics, Dharampeth M P Deo Memorial Science College, \\ Nagpur 440 010, India \\ ${ }^{2}$ Post Graduate Department of Mathematics, Nagpur University, Nagpur 440010 , \\ India \\ e-mail: kcdeshmukh2000@rediffmail.com
}

MS received 25 March 2004; revised 9 March 2005

\begin{abstract}
In this paper, we develop an integral transform to determine temperature distribution in a thin circular plate, subjected to a partially distributed and axisymmetric heat supply on the curved surface, and study the thermal deformation. The results, obtained in series form in terms of Bessel's functions, are illustrated numerically.
\end{abstract}

Keywords. Transient problem; thermal deformation; heat conduction problem.

\section{Introduction}

Thermal deflections of axisymmetrically heated circular plates in the case of fixed and simply supported edges have been considered by Boley \& Weiner (1960). Roy Choudhuri (1973) discussed the normal deflection of a thin clamped circular plate due to ramp-type heating of a concentric circular region of the upper face and the lower face of the plate kept at zero temperature, with the circular edge being thermally insulated. Noda et al (1997) discussed the transient thermoelastoplastic bending problem making use of the strain increment theorem and determined the temperature field and thermoelastic deformation for the heating and cooling processes in thin circular plates, subjected to partially distributed and axisymmetric heat supply on the upper face. Deshmukh \& Khobragade (2002) determined quasi-static thermal deflections in thin circular plates subjected to arbitrary initial temperatures on the lower face with the upper face at zero temperature and the fixed circular edge thermally insulated. Here, we extend the work of Noda et al (1997) and study the heat conduction problem to determine the temperature distribution and discuss the thermoelastic deformation in thin circular plates subjected to partially distributed and axisymmetric heat supply on the outer curved surface. The results are illustrated numerically. The results presented here will be more useful in engineering problems, particularly in machines subjected to heating and cooling. 


\section{Analysis}

\subsection{Transient heat conduction problem}

We consider a thin circular plate of radius $a$ and thickness $h$. The initial temperature of the plate is the same as the temperature of the surrounding medium, which is kept constant. From time $t=0$ to $t=t_{0}$, the plate is subjected to a partially distributed and axisymmetric heat supply $Q_{0} f(z)$ on the curved surface $(r=a)$. After time $t_{0}$, the heat supply is removed and the plate is cooled by the surrounding medium. For heating processes, the heat conduction equation, the initial condition, and the boundary conditions are given, respectively, as

$$
\begin{aligned}
& \frac{\partial^{2} T}{\partial r^{2}}+\frac{1}{r} \frac{\partial T}{\partial r}+\frac{\partial^{2} T}{\partial z^{2}}=\frac{1}{\kappa} \frac{\partial T}{\partial t}, \\
& \left.T(r, z, t)\right|_{t=0}=0, \\
& \left.T(r, z, t)\right|_{r=a}=-\left(Q_{0} / \lambda\right) f(z), \quad 0 \leq t \leq t_{0}, \\
& \left.T(r, z, t)\right|_{z=-h / 2}=0, \\
\text { and } \quad & \left.T(r, z, t)\right|_{z=h / 2}=0,
\end{aligned}
$$

where $\kappa$ and $\lambda$ are the thermal diffusivity and the thermal conductivity respectively.

On the other hand, for the cooling process, the temperature distribution $T^{\prime}(r, z, t)$ satisfies the equation

$$
\frac{\partial^{2} T^{\prime}}{\partial r^{2}}+\frac{1}{r} \frac{\partial T^{\prime}}{\partial r}+\frac{\partial^{2} T^{\prime}}{\partial z^{2}}=\frac{1}{\kappa} \frac{\partial T^{\prime}}{\partial t},
$$

with the initial and the boundary conditions given by

$$
\begin{aligned}
& \left.T^{\prime}(r, z, t)\right|_{t=t_{0}}=T\left(r, z, t_{0}\right), \\
& \left.T^{\prime}(r, z, t)\right|_{r=a}=0, \quad t \geq t_{0}, \\
& \left.T^{\prime}(r, z, t)\right|_{z=-h / 2}=0 \\
\text { and } \quad & \left.T^{\prime}(r, z, t)\right|_{z=h / 2}=0 .
\end{aligned}
$$

Equations (1) to (10) constitute the transient heat conduction problem for heating and cooling processes.

\section{Solution of the problem}

3.1 Determination of the temperatures $T(r, z, t)$ and $T^{\prime}(r, z, t)$

On applying the finite Hankel and the finite Fourier transforms to (1) to (10) and then using their inversions, one obtains the expressions of the temperature distributions $T(r, z, t)$ and $T^{\prime}(r, z, t)$ for the heating and the cooling processes respectively, as

$$
T(r, z, t)=\frac{-2(2)^{1 / 2} Q_{0}}{a \lambda(h)^{1 / 2}} \sum_{n=1}^{\infty} \sum_{m=1}^{\infty} \frac{\xi_{n} \bar{f}\left(\lambda_{m}\right) J_{0}\left(\xi_{n} r\right) \sin \left(\lambda_{m} z\right) \cdot\left[1-e^{-\kappa\left(\xi_{n}^{2}+\lambda_{m}^{2}\right) t}\right]}{\left(\xi_{n}^{2}+\lambda_{m}^{2}\right) J_{1}\left(\xi_{n} a\right)}
$$


and

$$
\begin{aligned}
T^{\prime}(r, z, t)= & \frac{-2(2)^{1 / 2} Q_{0}}{a \lambda(h)^{1 / 2}} \sum_{n=1}^{\infty} \sum_{m=1}^{\infty} \\
& \times \frac{\xi_{n} \bar{f}\left(\lambda_{m}\right) J_{0}\left(\xi_{n} r\right) \sin \left(\lambda_{m} z\right)\left[1-e^{\left.-\kappa\left(\xi_{n}^{2}+\lambda_{m}^{2}\right) t_{0}\right)}\right]\left[e^{-\kappa\left(\xi_{n}^{2}+\lambda_{m}^{2}\right)\left(t-t_{0}\right)}\right]}{\left(\xi_{n}^{2}+\lambda_{m}^{2}\right) J_{1}\left(\xi_{n} a\right)}
\end{aligned}
$$

where $\xi_{n}$ is the $n$th positive root of the transcendental equation

$$
J_{0}(\xi a)=0,
$$

$\lambda_{m}$ is the $m$ th positive root of the transcendental equation,

$$
\begin{aligned}
& \sin (\lambda h / 2)=0, \\
& \bar{f}\left(\lambda_{m}\right)=\int_{-h / 2}^{h / 2} f(z) \cdot K\left(\lambda_{m}, z\right) d z,
\end{aligned}
$$

$K\left(\lambda_{m}, z\right)$ is the kernel for the finite Fourier transform defined by

$$
K\left(\lambda_{m}, z\right)=\left((2)^{1 / 2} /(h)^{1 / 2}\right) \sin \left(\lambda_{m} z\right)
$$

and $K_{0}\left(\xi_{n}, r\right)$ is the kernel for the finite Hankel transform defined by

$$
K_{0}\left(\xi_{n}, r\right)=\left((2)^{1 / 2} / a\right)\left\{J_{0}\left(\xi_{n} r\right)\right\} /\left\{J_{0}^{\prime}\left(\xi_{n} a\right)\right\} .
$$

Using the result (11) into (12), one obtains

$$
T^{\prime}(r, z, t)=T(r, z, t)-T\left(r, z, t-t_{0}\right) .
$$

Equation (18) represents the relation between the temperatures in the heating and cooling processes.

Further, equation (11) for the heating and the cooling processes together can be rewritten as

$$
T(r, z, t)=\frac{-2(2)^{1 / 2} Q_{0}}{a \lambda(h)^{1 / 2}} \sum_{n=1}^{\infty} \sum_{m=1}^{\infty} A\left(\xi_{n}, \lambda_{m}, t\right) \bar{f}\left(\lambda_{m}\right) J_{0}\left(\xi_{n} r\right) \sin \left(\lambda_{m} z\right),
$$

where

$$
\begin{aligned}
A\left(\xi_{n}, \lambda_{m}, t\right) & =\left\{\xi_{n}\left[1-e^{-\kappa\left(\xi_{n}^{2}+\lambda_{m}^{2}\right) t}\right]\right\} /\left(\xi_{n}^{2}+\lambda_{m}^{2}\right) J_{1}\left(\xi_{n} a\right) \\
& =A_{m n}\left(\xi_{n}, \lambda_{m}, t\right), \quad\left(0 \leq t \leq t_{0}\right)
\end{aligned}
$$

and

$$
\begin{aligned}
A\left(\xi_{n}, \lambda_{m}, t\right) & =\left\{\xi_{n}\left[1-e^{-\kappa\left(\xi_{n}^{2}+\lambda_{m}^{2}\right) t_{0}}\right] \cdot e^{-\kappa\left(\xi_{n}^{2}+\lambda_{m}^{2}\right)\left(t-t_{0}\right)}\right\} /\left(\xi_{n}^{2}+\lambda_{m}^{2}\right) J_{1}\left(\xi_{n} a\right) \\
& =A_{m n}\left(\xi_{n}, \lambda_{m}, t_{0}\right) e^{-k\left(\xi_{n}^{2}+\lambda_{m}^{2}\right)\left(t-t_{0}\right)}, \quad\left(t \geq t_{0}\right)
\end{aligned}
$$




\section{Thermoelastic problem}

\subsection{Derivation of basic equations}

To derive the basic equations that govern the thermoelastic behaviour of the plate, assume that the plate is very thin. Therefore, the assumption that the plane perpendicular to the neutral plane $(z=0)$ before deformation, remains the one perpendicular to it after deformation. The differential equation satisfied by "the resultant force function" $F$ is

$$
\frac{\mathrm{d}}{\mathrm{d} r}\left[\nabla^{2} F\right]=-\frac{\mathrm{d} N_{T}}{\mathrm{~d} r},
$$

where $\nabla^{2}$ denotes the two-dimensional and axisymmetric Laplace operator in cylindrical coordinate defined as

$$
\nabla^{2}=\left(\mathrm{d}^{2} / \mathrm{d} r^{2}\right)+(1 / r)(\mathrm{d} / \mathrm{d} r) .
$$

The thermal resultant force $N_{T}$ is defined as

$$
N_{T}=\int_{-h / 2}^{h / 2} \alpha E T \mathrm{~d} z
$$

$\alpha$ and $E$ are the coefficients of the linear thermal expansion and Young's modulus respectively.

For in-plane deformation, the boundary conditions are given by

$$
F=\mathrm{d} F / \mathrm{d} r=0, \text { at } r=a .
$$

The differential equation satisfied by "the deflection" $\omega(r, t)$ is

$$
D(\mathrm{~d} / \mathrm{d} r)\left[\nabla^{2} \omega\right]=[1 /(1-v)]\left(\mathrm{d} M_{T} / \mathrm{d} r\right),
$$

where $D$ is the flexural rigidity of the plate denoted by

$$
D=E h^{3} /\left[12\left(1-v^{2}\right)\right],
$$

$v$ is Poisson's ratio and the thermal resultant moment $M_{T}$ is defined as

$$
M_{T}=\int_{-h / 2}^{h / 2} \alpha E T z \mathrm{~d} z
$$

For out-of-plane deformation, the boundary conditions are given as

$$
\omega=0 \text {, at } r=0,
$$

and

$$
D\left(\left(\mathrm{~d}^{2} \omega / \mathrm{d} r^{2}\right)+(\nu / r)(\mathrm{d} \omega / \mathrm{d} r)\right)=-M_{T} /(1-v), \text { at } r=a .
$$

Equations (22) to (30) constitute the formulation of the thermoelastic problem. 
4.2 Determination of the force component, $F(r, t)$

Substituting the value of $T(r, z, t)$ from (19) into (24), we obtain

$$
N_{T}=0
$$

Solving (22) by using the value of $N_{T}$ from (31) under the conditions (25), we obtain

$$
F=F(r, t)=0 .
$$

\subsection{Determination of the deflection, $\omega(r, t)$}

Substituting the value of $T(r, z, t)$ from (19) into (28), we obtain

$$
M_{T}=\frac{2(2)^{1 / 2} Q_{0} \alpha E(h)^{1 / 2}}{a \lambda} \sum_{n=1}^{\infty} \sum_{m=1}^{\infty} \frac{A\left(\xi_{n}, \lambda_{m}, t\right) \bar{f}\left(\lambda_{m}\right) \cos \left(\lambda_{m} h / 2\right) J_{0}\left(\xi_{n} r\right)}{\lambda_{m}} .
$$

From (32), we can solve (26) under the conditions (29) and (30). The deflection $\omega(r, t)$ is given as

$$
\begin{aligned}
\omega(r, t)= & \frac{2(2)^{1 / 2} Q_{0} \alpha E(h)^{1 / 2}}{D(1-v) a \lambda} \sum_{n=1}^{\infty} \sum_{m=1}^{\infty} \frac{A\left(\xi_{n}, \lambda_{m}, t\right) \bar{f}\left(\lambda_{m}\right) \cos \left(\lambda_{m} h / 2\right)}{\xi_{n} \lambda_{m}} \\
& \times\left[J_{0}\left(\xi_{n} r\right)-\frac{r^{2} \xi_{n}}{2(1+v)}\left\{\left(1-\xi_{n}\right) J_{0}\left(\xi_{n} a\right)+\left(\frac{1-v}{a}\right) J_{1}\left(\xi_{n} a\right)\right\}-1\right] .
\end{aligned}
$$

For the variation of angle with time, define the angle $\theta$ as

$$
\theta=-\mathrm{d} \omega / \mathrm{d} r
$$

Substituting the value of $\omega(r, t)$ from (34) into (35), one obtains

$$
\begin{aligned}
\theta= & \frac{2(2)^{1 / 2} Q_{0} \alpha E(h)^{1 / 2}}{D\left(1-v^{2}\right) a^{2} \lambda} \sum_{n=1}^{\infty} \sum_{m=1}^{\infty}\left[\frac{A\left(\xi_{n}, \lambda_{m}, t\right) \bar{f}\left(\lambda_{m}\right) \cos \left(\lambda_{m} h / 2\right)}{\lambda_{m}}\right] \\
& \times\left[a(1+v) J_{1}\left(\xi_{n} r\right)+r\left\{a\left(1-\xi_{n}\right) J_{0}\left(\xi_{n} a\right)+(1-v) J_{1}\left(\xi_{n} a\right)\right\}\right] .
\end{aligned}
$$

\section{Special case}

Set

$$
f(z)=\left(4 z^{2}-h^{2}\right) z
$$

Apply the finite Fourier transform to the equation (37), one obtains

$$
\bar{f}\left(\lambda_{m}\right)=\left[24(2)^{1 / 2}(h)^{1 / 2} \cos \left(\lambda_{m} h / 2\right)\right] / \lambda_{m}^{3} .
$$


Putting the value of $\bar{f}\left(\lambda_{m}\right)$ from (38) into (19), (34) and (36), temperature, deflection and angle respectively, are obtained as

$$
\begin{aligned}
T(r, z, t)= & \frac{-96 Q_{0}}{a \lambda} \sum_{n=1}^{\infty} \sum_{m=1}^{\infty} \frac{A\left(\xi_{n}, \lambda_{m}, t\right) \cdot \cos \left(\lambda_{m} h / 2\right) \cdot J_{0}\left(\xi_{n} r\right) \cdot \sin \left(\lambda_{m} z\right)}{\lambda_{m}^{3}}, \\
\omega(r, t)= & \frac{96 Q_{0} \alpha E h}{D(1-v) a \lambda} \sum_{n=1}^{\infty} \sum_{m=1}^{\infty} \frac{A\left(\xi_{n}, \lambda_{m}, t\right) \cdot \cos \left(\lambda_{m} h / 2\right)}{\xi_{n} \lambda_{m}^{4}} \\
& \times\left[J_{0}\left(\xi_{n} r\right)-\frac{r^{2} \xi_{n}}{2(1+v)}\left\{\left(1-\xi_{n}\right) J_{0}\left(\xi_{n} a\right)+\left(\frac{1-v}{a}\right) J_{1}\left(\xi_{n} a\right)\right\}-1\right] .
\end{aligned}
$$

and

$$
\begin{aligned}
\theta= & \frac{96 Q_{0} \alpha E h}{D\left(1-v^{2}\right) a^{2} \lambda} \sum_{n=1}^{\infty} \sum_{m=1}^{\infty} \frac{A\left(\xi_{n}, \lambda_{m}, t\right) \cdot \cos \left(\lambda_{m} h / 2\right)}{\lambda_{m}^{4}} \\
& \times\left[a(1+v) J_{1}\left(\xi_{n} r\right)+r\left\{a\left(1-\xi_{n}\right) J_{0}\left(\xi_{n} a\right)+(1-v) J_{1}\left(\xi_{n} a\right)\right\}\right] .
\end{aligned}
$$

\section{Numerical results}

The numerical calculations have been carried out for a steel (SN50C) plate with the properties $\kappa=15.9 \times 10^{-6}\left[\mathrm{~m}^{2} \mathrm{~s}^{-1}\right], v=0 \cdot 281, \lambda=59 \cdot 0\left[\mathrm{Wm}^{-1} \mathrm{~K}^{-1}\right]$. Setting

$$
\alpha=\frac{-96 Q_{0}}{a \lambda}, \beta=\frac{96 Q_{0} \alpha E h}{D(1-v) a \lambda}, \gamma=\frac{96 Q_{0} \alpha E h}{D\left(1-v^{2}\right) a^{2} \lambda},
$$

with the radius $a=5$ and thickness $h=1$ into (39), (40) and (41), one gets the expressions for temperature distribution, thermal deflection and angle respectively as,

$$
\begin{aligned}
\frac{T}{\alpha}= & \sum_{n=1}^{\infty} \sum_{m=1}^{\infty} \frac{A\left(\xi_{n}, \lambda_{m}, t\right) \cdot \cos \left(\lambda_{m} / 2\right) \cdot J_{0}\left(\xi_{n} r\right) \cdot \sin \left(\lambda_{m} z\right)}{\lambda_{m}^{3}} \\
\frac{\omega}{\beta}= & \sum_{n=1}^{\infty} \sum_{m=1}^{\infty} \frac{A\left(\xi_{n}, \lambda_{m}, t\right) \cdot \cos \left(\lambda_{m} h / 2\right)}{\xi_{n} \lambda_{m}^{4}} \\
& \times\left[J_{0}\left(\xi_{n} r\right)-\frac{r^{2} \xi_{n}}{2(1+v)}\left\{\left(1-\xi_{n}\right) J_{0}\left(5 \xi_{n}\right)+\left(\frac{1-v}{5}\right) J_{1}\left(5 \xi_{n}\right)\right\}-1\right]
\end{aligned}
$$

and

$$
\begin{aligned}
\frac{\theta}{\gamma}= & \sum_{n=1}^{\infty} \sum_{m=1}^{\infty} \frac{A\left(\xi_{n}, \lambda_{m}, t\right) \cdot \cos ^{2}\left(\lambda_{m} h / 2\right)}{\lambda_{m}^{4}} \\
& \times\left[5(1+v) J_{1}\left(\xi_{n} r\right)+r\left\{5\left(1-\xi_{m}\right) J_{0}\left(5 \xi_{n}\right)+(1-v) J_{1}\left(5 \xi_{n}\right)\right\}\right]
\end{aligned}
$$




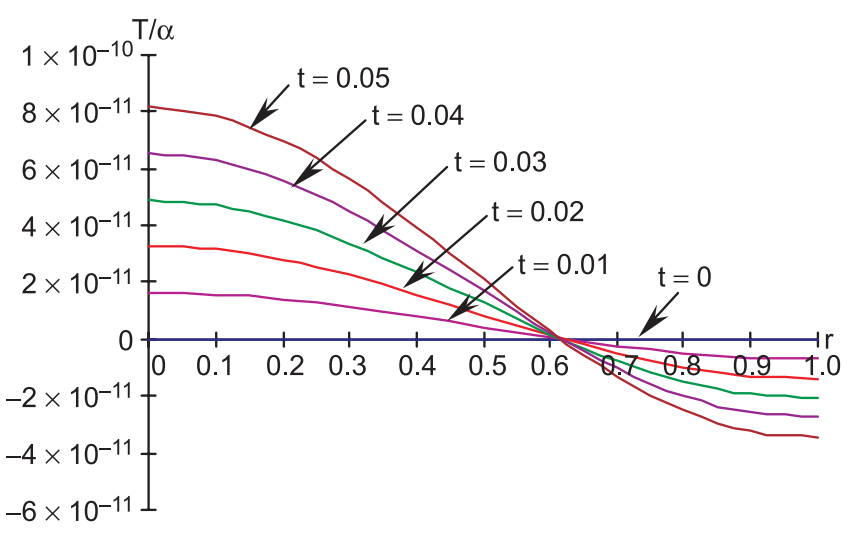

Figure 1. Temperature distribution $T / \alpha$ at $t=0,0.01, \ldots, 0.05$ for fixed $z=0.5\left(0 \leq t \leq t_{0}\right)$.

where $\xi=0.4809, \xi_{2}=1.1040, \xi_{3}=1.7307, \xi_{4}=2.3583, \xi_{5}=2.9861, \xi_{6}=3.6142$ are the positive roots of the transcendental equation $J_{0}(\xi a)=0$ which takes the form $J_{0}(5 \xi)=0$ for $a=5$ (Özisik 1968) and $\lambda_{1}=6 \cdot 28, \lambda_{2}=12.56, \lambda_{3}=18 \cdot 84, \lambda_{4}=25 \cdot 12, \lambda_{5}=$ 31.40 are positive roots of the transcendental equation $\sin (\lambda h / 2)=0$, which takes the form $\sin (\lambda / 2)=0$ for $h=1$ (Özisik 1968). These values are used in (42), (43) and (44) to illustrate the temperature distribution, thermal deflection and angle of variation numerically in the heating as well as the cooling process with the help of a computer and variations, as shown in figures $1-6$.

Figure 1 indicates that temperature increases in the heating process $\left(0 \leq t \leq t_{0}\right)$ while figure 2 shows that temperature decreases after removing the heat supply in the cooling process $\left(t \geq t_{0}\right)$. Figure 3 shown that deflection increases in the heating process $\left(0 \leq t \leq t_{0}\right)$ while figure 4 shows that deflection decreases after removing the heat supply in the cooling process $\left(t \geq t_{0}\right)$. In figure 5, the angle of variation increases during the heating process $\left(0 \leq t \leq t_{0}\right)$ while figure 6 shows that the angle of variation decreases after removing the heat supply in the cooling process $\left(t \geq t_{0}\right)$.

\section{Conclusions}

In this article, we extend the problem studied by Noda et al (1997) and study the heat conduction problem of a thin circular plate due to the partially distributed and axisymmetric heat

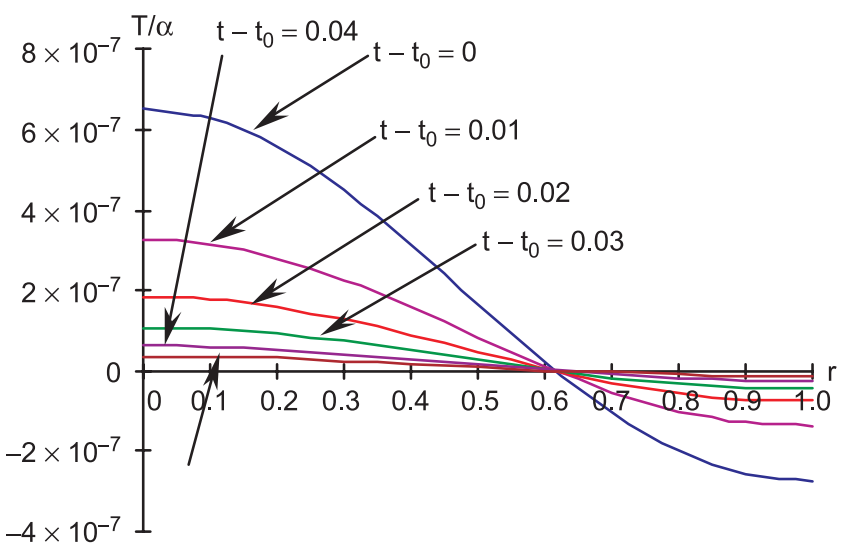

Figure 2. Distribution of temperature $T / \alpha$ at $t-t_{0}=0$, $0.01, \ldots, 0.05$ for fixed $z=0.5$ $\left(t \geq t_{0}\right)$. 


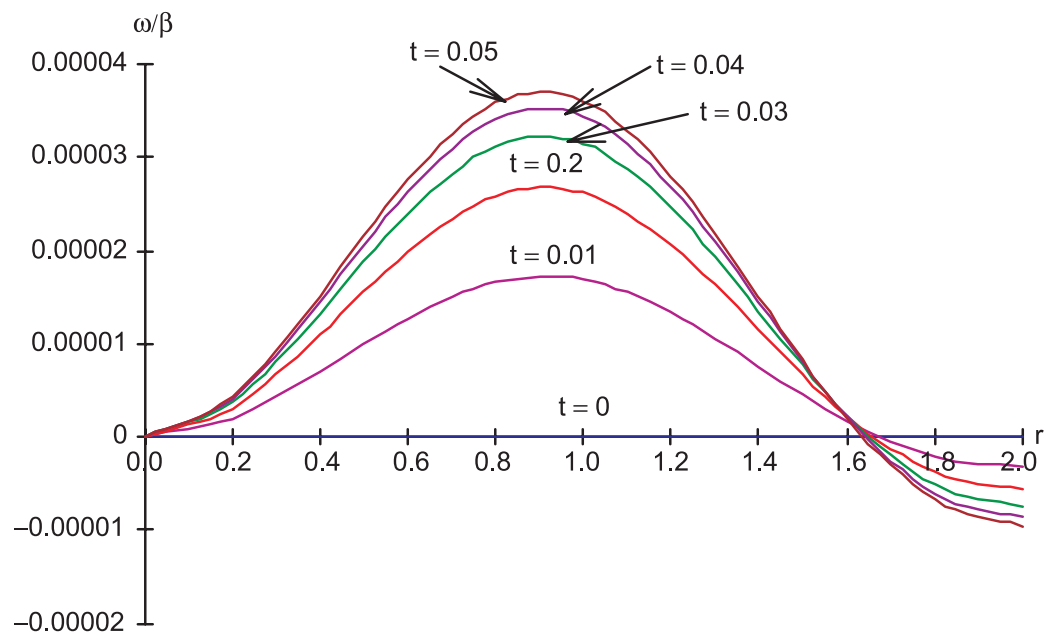

Figure 3. Distribution of deflection $\omega / \beta$ at $t=0,0 \cdot 01, \ldots, 0 \cdot 05\left(0 \leq t \leq t_{0}\right)$.

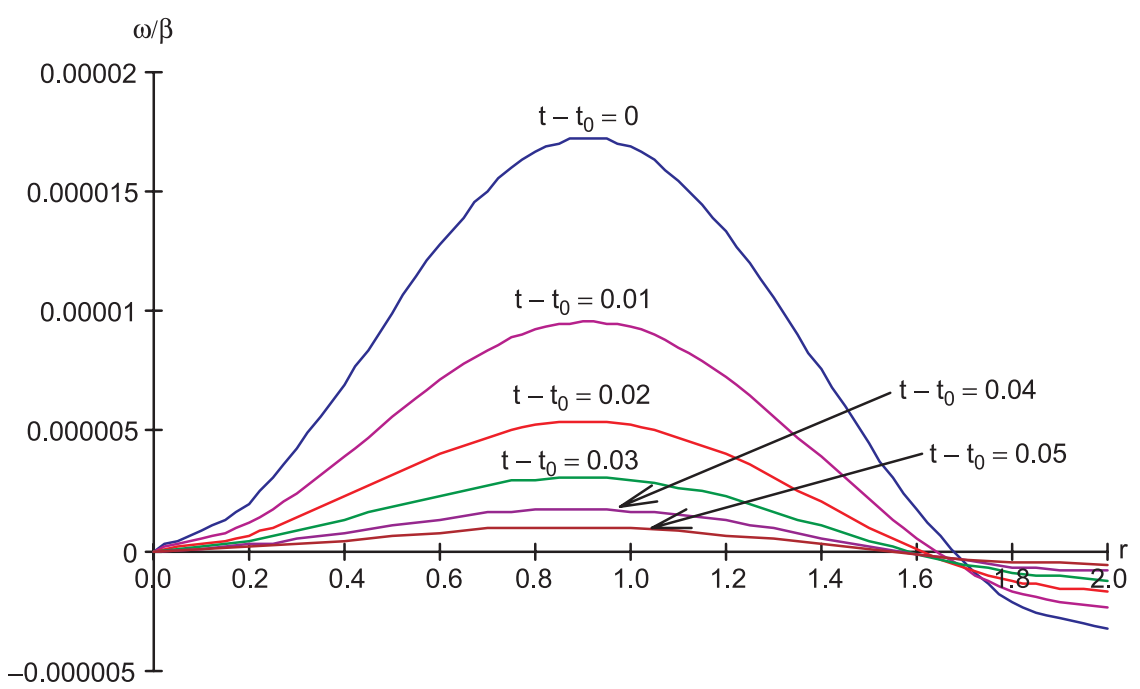

Figure 4. Distribution of deflection $\omega / \beta$ at $t-t_{0}=0,0 \cdot 01, \ldots, 0 \cdot 05\left(0 \geq t_{0}\right)$.

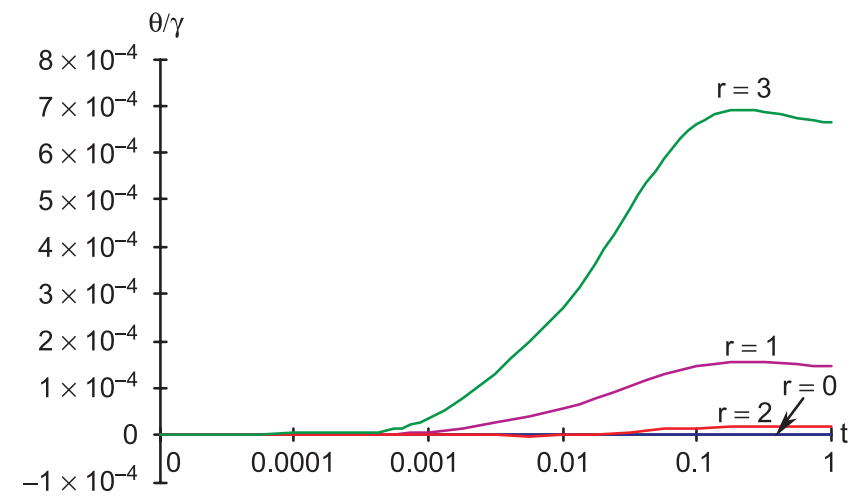

Figure 5. Variation of angle with time at $r=0,1,2,3\left(0 \leq t \leq t_{0}\right)$. 


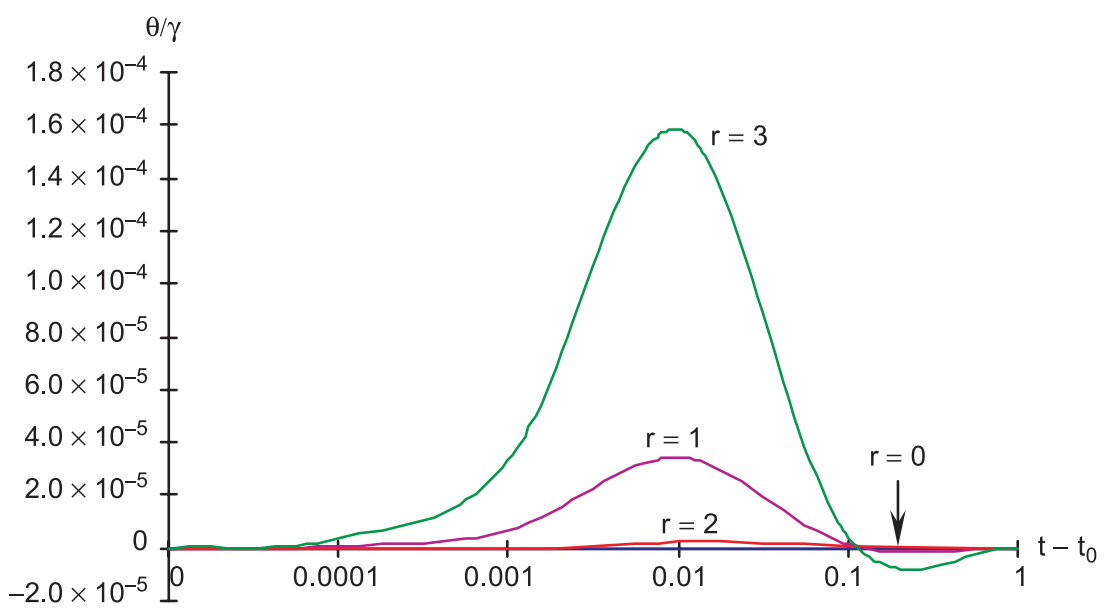

Figure 6. Variation of angle with time at $r=0,1,2,3\left(t \geq t_{0}\right)$.

supply on the outer curved surface. We find the temperature field analytically by introducing the methods of the finite Fourier and the finite Hankel transforms and determine the thermal deformation by using the temperature field for the heating and cooling processes. Considering steel, we obtain expressions for the temperature distribution, thermal deflection and angle of variation in the heating as well as cooling media, which are illustrated numerically and shown in figures $1-6$.

The force component $F(r, t)$ reduces to zero due to the absence of plane strain on the circular region of the plate. This type of problem is mainly applicable in the engineering area, particularly in machines subjected to heating and cooling. Any particular case of special interest can be described by assigning suitable values to the parameters and functions in (42), (43) and (44).

The authors express their sincere thanks to Dr P C Wankhede for help in preparing this paper and also thank the U.G.C. for financial support. The authors express their sincere thanks to the referees for suggesting the changes in an earlier version of this manuscript.

\section{References}

Boley B A, Weiner J H 1960 Theory of thermal stresses (New York: Wiley)

Deshmukh K C, Khobragade N L 2002 Quasi-static thermal deflection in a thin circular plate. Far East J. Appl. Math. 9(3): 171-179

Noda N, Ishihara M, Tanigawa Y, Kawamura R 1997 Theoretical analysis of thermoelastoplastic deformation of a circular plate due to a partially distributed heat supply. J. Thermal Stresses 20: 203-225

Özisik M N 1968 Boundary value problem of heat conduction. Tables for the roots of transcendental equations (Scranton, PA: Int. Text Book Co.) pp 481-492

Roy Choudhuri S K 1973 A note on the quasi-static thermal deflection o a thin clamped circular plate due to rap-type heating of a concentric circular region of the upper face. J. Franklin Inst. 296: 203-225 